\title{
Bacterial immune modulation of the cytokine response elicited by gliadin in an in vitro model ofceliac disease
}

\author{
T. Pozo-Rubio ${ }^{1}$, A. Marcos ${ }^{1}$, F. Koning ${ }^{2}$, J. R. Mujico ${ }^{1}$, Y. Sanz ${ }^{3}$ and E. Nova ${ }^{1}$ \\ ${ }^{1}$ Immunonutrition group, ICTAN, CSIC, Madrid, Spain, ${ }^{2}$ Department of Immunohematology and Blood Transfusion, LUMC, \\ Leiden, The Netherlands, ${ }^{3}$ Microbial Ecophysiology and Nutrition Group, IATA, CSIC, Valencia, Spain
}

\begin{abstract}
Gluten peptides have been shown to induce cytotoxicity and IL-15 production even in in vitro studies with cell lines and biopsies of healthy subjects ${ }^{(1,2)}$. We hypothesized that the inflammatory milieu caused by gluten antigens might be counteracted by certain species or strains of the commensal intestinal microbiota in interaction with the immune system. The aim of the present study was to evaluate the effect of different bacterialstrains from breastfed (BF) and formula-fed (FF) infants at risk ofceliac disease on cytokine production and $\mathrm{T}$ cell proliferationin vitro. Two combinations of predominant bacteria from fecal samples ofBF (Escheriquia coli (51.6\%), Lactobacillus casei $(19.4 \%)$, and Bifidobacterium breve (29\%)) and FF (Klebsiella pneumoniae (44.1\%), Lactobacillus rhamnosus (29.4\%), and Bifidobacterium longum (26.5\%)) infants were used. Caco-2 monolayers grown in a transwell cell-culture system (12mm inserts (Millipore)) were challenged by apical addition of $2 \times 10^{6} \mathrm{cfu} /$ insert ofbacteria. PBMCs $\left(1 \times 10^{6}\right.$ cells/well $)$ and gluten specific $\mathrm{T}$ cell clones $\left(1 \times 10^{5}\right.$ cells/well $)$ from HLA-DQ2 patients were added in the basal compartment of the culture well for a 12-hour incubation.Gliadin $(7.5 \mathrm{mg} / \mathrm{mL})$ was also added at the same time in the basal or apical compartment. Thereafter, further 36 hours incubation was allowed after disassembly of the system in order to measure the cytokine production by the sensitized Caco-2, and cytokine production and proliferation by $\mathrm{T}$ cell clones separately.TNF- $\alpha$, IL-6, IL-1 $\beta$, and IL- 8 cytokines were measured in Caco- 2 cells basolateral medium, and TNF- $\alpha$, IL-6, IL-1 $\beta$, and IL-10 cytokines were measured in T cells supernatant by Cytometric Bead Array Flex sets (BD Biosciences) and analyzed by flow cytometry. $\mathrm{T}$ cell proliferation was measured by quantification of $\mathrm{H}^{3}$-thymidine incorporation. Gliadin in the apical compartment did not show any effect on $\mathrm{T}$ cell proliferation and cytokine production. In the basal compartment Gliadin plus breastfed mixture (Gli-BF) showed a lower $\mathrm{T}$ cell proliferation than gliadin plus formula-fed mixture (Gli-FF) and gliadin alone. IL-10 in T cell supernatants, and IL-1 $\beta$ in Caco-2 supernatants were below the minimum detectable concentration in all conditions.Gliadin, Gli-BF and Gli-FF induced cytokine secretion compared to control without gliadin, however the BF mixture inhibited the production of IL-6 and TNF- $\beta$ compared to Gli-FF and gliadin alone. When the bacteria combination plus gliadin were cultured in direct contact with the PBMC and $\mathrm{T}$ cells and no Caco-2 cells, the inhibition of cytokine production by theBF bacteria combination compared to gliadin alone was also observed, while FF combination did not reduce cytokine production and proliferation.We suggest that certain bacteria combinations, as observed with those more prevalent in feces from breast fed infants, might attenuated the effect of gliadin onT-cell proliferation and cytokine production.
\end{abstract}

This work was supported by grants AGL2007-66126-C03-01/ALI and 03/ALI from the Spanish Ministry of Science and Innovation. T. Pozo-Rubio is recipient of a JAE-CSIC grant co-funded by European Social Fund.

1. Bernardo D, Garrote JA, Fernandez-Salazar L et al. (2007) Gut 56, 889-890.

2. Elli L, Dolfini E \& Bardella MT (2003) Toxicology Letters 146, 1-8. 